\title{
Der tierische Bewuchs an Helgoländer Seetonnen
}

\author{
Von H. Caspers \\ Aus der Biologischen Anstalt Helgoland und der Hydrobiologischen Abteilung \\ des Zoologischen Staatsinstitutes und Zoologischen Museums Hamburg
} (Mit 9 Abbildungen im Text)

\section{Einleitung}

Der tierische Bewuchs an Seetonnen ist bisher erst in wenigen Fällen Gegenstand einer Untersuchung gewesen. Nach der klassischen Arbeit von KIRCHENPAUER (1862) über "Die Seetonnen der Elbmündung“ wandte sich die Aufmerksamkeit weitgehend dem - vorwiegend tierischen - Bewuchs an Seeschiffen zu, da hier die Ergebnisse auch für die Praxis von größtem Wert sind. Besonders sind die Arbeiten von Hentschel (1923, 1924), NEU und VIsscher (1930) (vgl. die Schriftenübersicht: NEU, 1933 b) zu nennen. Die biologischen Verhältnisse sind hier aber entsprechend dem großen Fahrbereich besonders bei Uberseeschiffen außerordentlich schwierig; auch stellen die sich ansetzenden Tiere eine Auslese dar, die durch Reibung bei der Fahrt, Salzgehalts- und Temperaturwechsel usw. gegeben ist. Wie Hentscher. eindeutig festgestellt hat, findet der Ansatz hier fast ausschließlich während der Liegezeit der Schiffe (Hafen oder Reede) statt. Für die Tonnen ist kennzeichnend, daß sie als ständig verankerte Schwimmkörper in dem sich auf ihnen bildenden Bewuchs die biologischen Verhältnisse am Ort widerspiegeln, wobei einmal die genaue zeitliche Datierung, zum anderen die exakte Untersuchungsmöglichkeit beim Aufholen gegeben ist.

Hentschel (1923, S. 218) gibt für die Untersuchung mariner "stereophiler" Tiere (und Pflanzen) drei Hauptschwierigkeiten an: 1. kann der Bewudhs selten vollständig an die Oberfläche gebracht werden (z. B. Felsen); 2. sind die Substrate zu unregelmäßig gestaltet, als daß es möglich wäre, den Bewuchs durch Stichproben quantitativ zu kennzeichnen; 3. sind an den Wohnstätten des Bewuchses (Ufernähe) die Lebensbedingungen außerordentlich mannigfaltig und zeitlich wechselnd. - Von diesen Schwierigkeiten (die neuerdings in Ufergebieten durch direkte Taucherbeobachtungen etwas behoben werden) fallen bei den Seetonnen die 1. und 2 . völlig weg, ja die Gleichheit des Substrates an regional verschiedenen und festbestimmten Punkten läßt die Tonnen als besonders geeignet für die Betrachtung der Probleme erscheinen. Zum dritten Punkt muß bemerkt werden, daß die Mannigfaltigkeit der Lebensbedingungen und ihr zeitlicher Wechsel unser besonderes ökologisches Interesse hat. Im übrigen sind die Tonnen in der. Umgebung Helgolands auch hierzu besonders geeignet, da sie eigentlich "Hochseetonnen" darstellen, deren Lage untereinander nur geringe Unterschiede der Außenfaktoren ergibt (im Vergleich zu Tonnen in Flußmündungen). Die Bearbeitung des Bewuchses an diesen im Meer an verschiedenen Stellen verankerten "Versuchsstationen" kann daher zu Ergebnissen führen, die auch zur Klärung der schwierigeren Verhältnisse an fahrenden Schiffen beitragen. Weiter sind die Beziehungen zu den sessilen Gemeinschaften der Meeresküsten 
zu untersuchen, wobei bei letzteren durch die Trodkenliegezeit bei Ebbe besondere ökologische Differenzierungen gegeben sind.

Außer den Beobachtungen an Hafenmolen, Brücken usw. (z. B. Coe, 1932; Caspers, 1941, 1949; KüHL, 1951) liegen Arbeiten von Fraser (1938) über Tonnen in der LiverpoolBucht und Milne (1940) über die Tonnen im Tamar-Aestuar vor (s. auch Orton, 1930). Ferner ist die Untersuchung des Elbfeuerschiffes "Elbe $2^{*}$ durch Nev (1933 a) zu nennen.

Die im näheren und weiteren Bereich des helgoländer Seegebietes untersuchten Tonnen sind in die Karte Abb. 1 eingetragen. Sie liegen einmal unmittelbar vor der Insel (vor allem in der Reede zwischen Hauptinsel und Düne und in der. Nord- und Südeinfahrt zur Reede und zum Hafen), zum anderen um das Hummerschutzgebiet (HSG-Tonnen) und um die Dünenriffe; weiter entfernte Tonnen kennzeichnen verschiedene Gründe und Bänke ( $z$. B. Loreleybank $7 \mathrm{Sm}$ und Steingrund bis $11 \mathrm{Sm}$ vor der Insel). Die Liegezeit der Tonnen ist sehr unterschiedlich, von 1-24 Monate. Abb. 2 bringt mit der Liste der untersuchten Tonnen die Liegezeit in graphischer Darstellung ${ }^{1}$ ); eine Reihe der Tonnen wurde nach mehreren aufeinander folgenden Liegezeiten untersucht.

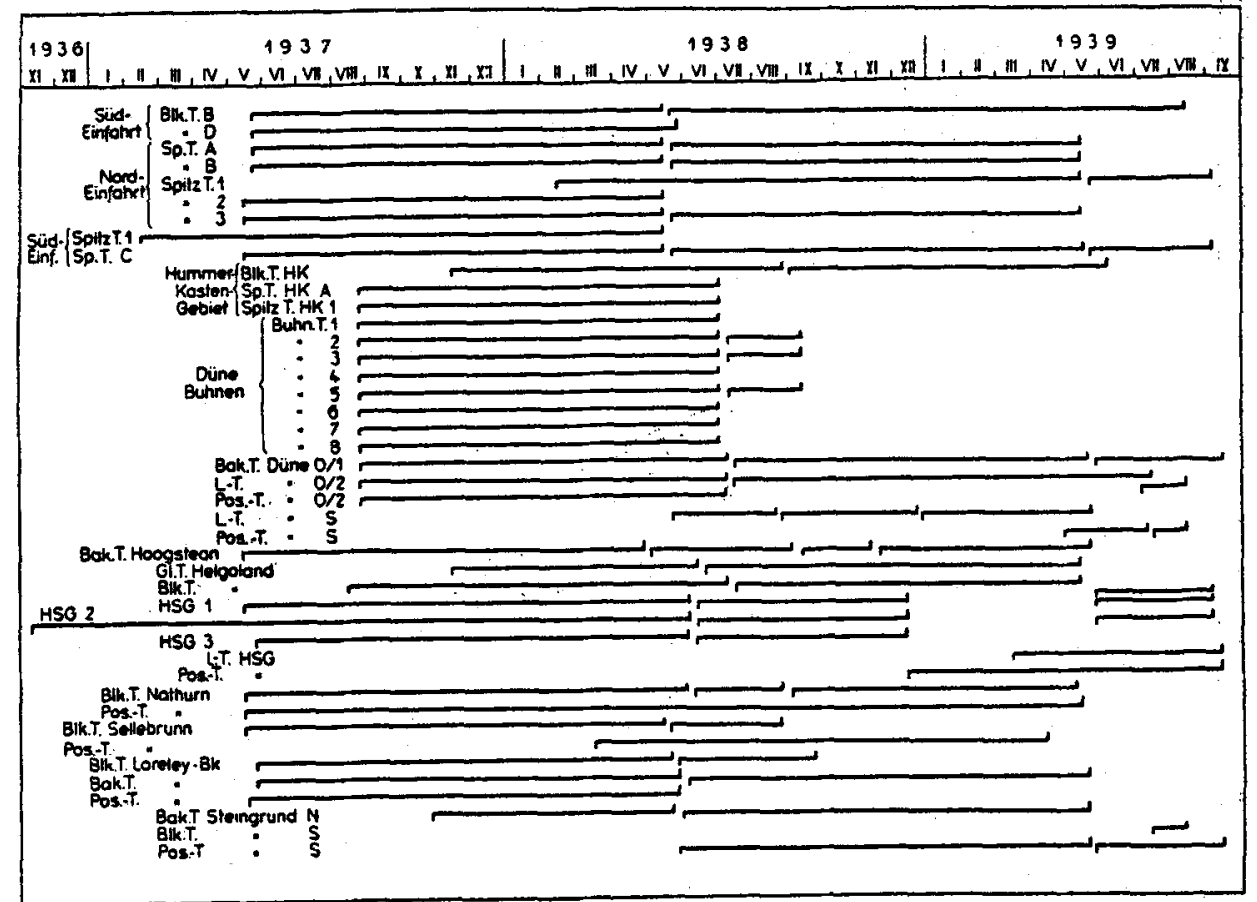

Abb. 2. Liste der untersuchten Tonnen mit graphischer Darstellung ihrer Liegezeiten 1936 bis 1939 (nur eingetragen die Liegezeiten, nach denen der Bewuchs untersucht wurde). Abkürzungen s. Anm.l.

Bei der Bearbeitung des helgoländer Tonnenbewuchses 1938-1939 sollte die gesamte Lebensgemeinschaft eine Behandlung finden. Die Untersuchung des Algenbewuchses hatte Herr Dr. K. BETh übernommen, dessen Darstellung der meinen über den tierischen Bewuchs parallel geht $\left.(1952)^{2}\right)$. Für die Bearbeitung der tierischen Lebensgemeinschaft sind neben den festsitzenden auch

1) Abkürzungen: Sp.T.: Spierentonne - Buhn.T.: Buhnentonne - L.T.: Leuchttonne Bak.T.: Bakentonne - Blk.T.: Blinktonne - Pos.T.: Positionstonne - SpitzT.: Spitztonne.

2) Siehe dort auch die Angaben über die Tonnentypen und ihre Größe. 


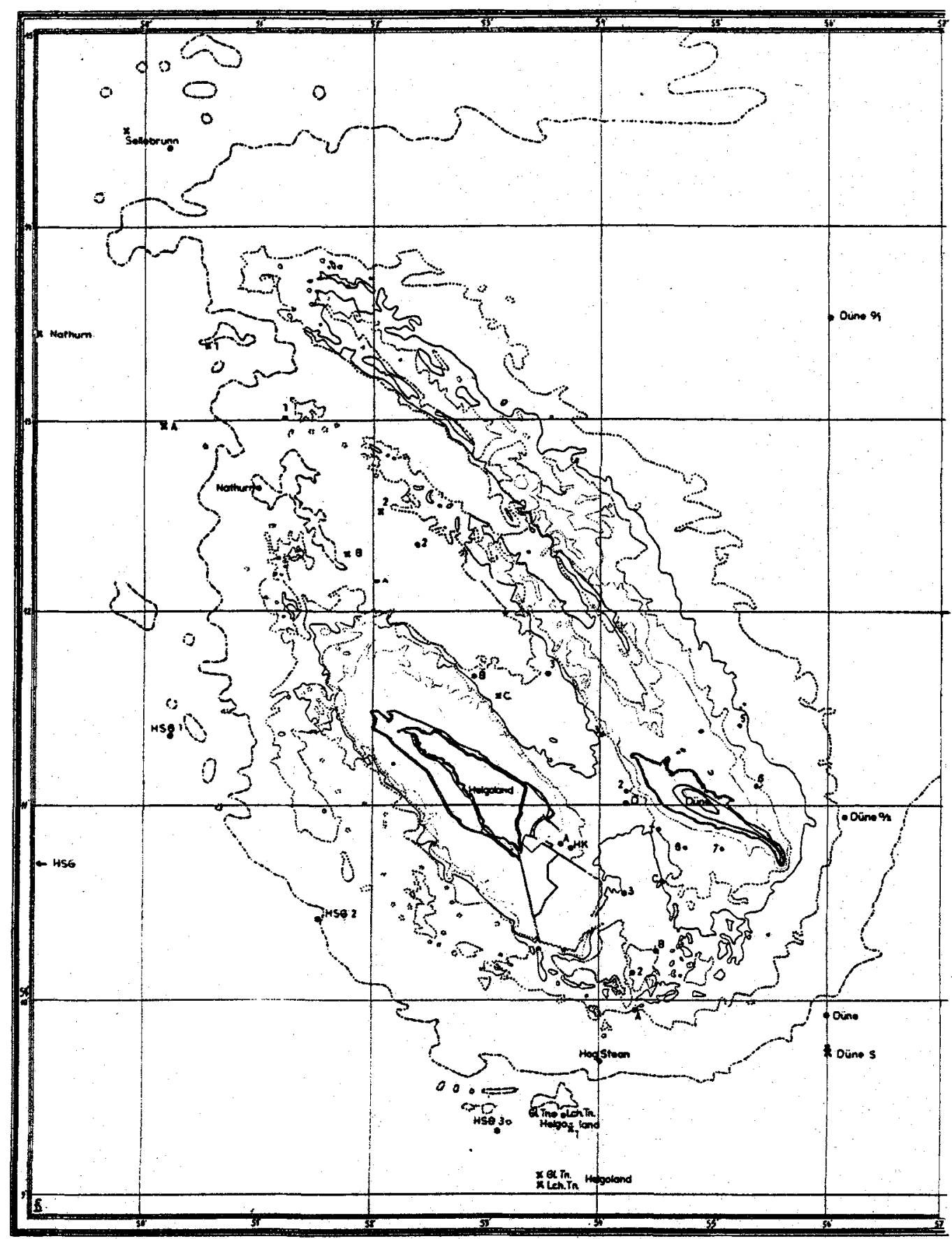

Abb. 1. Karte der helgoländer Umgebung mit der Betonnung. 


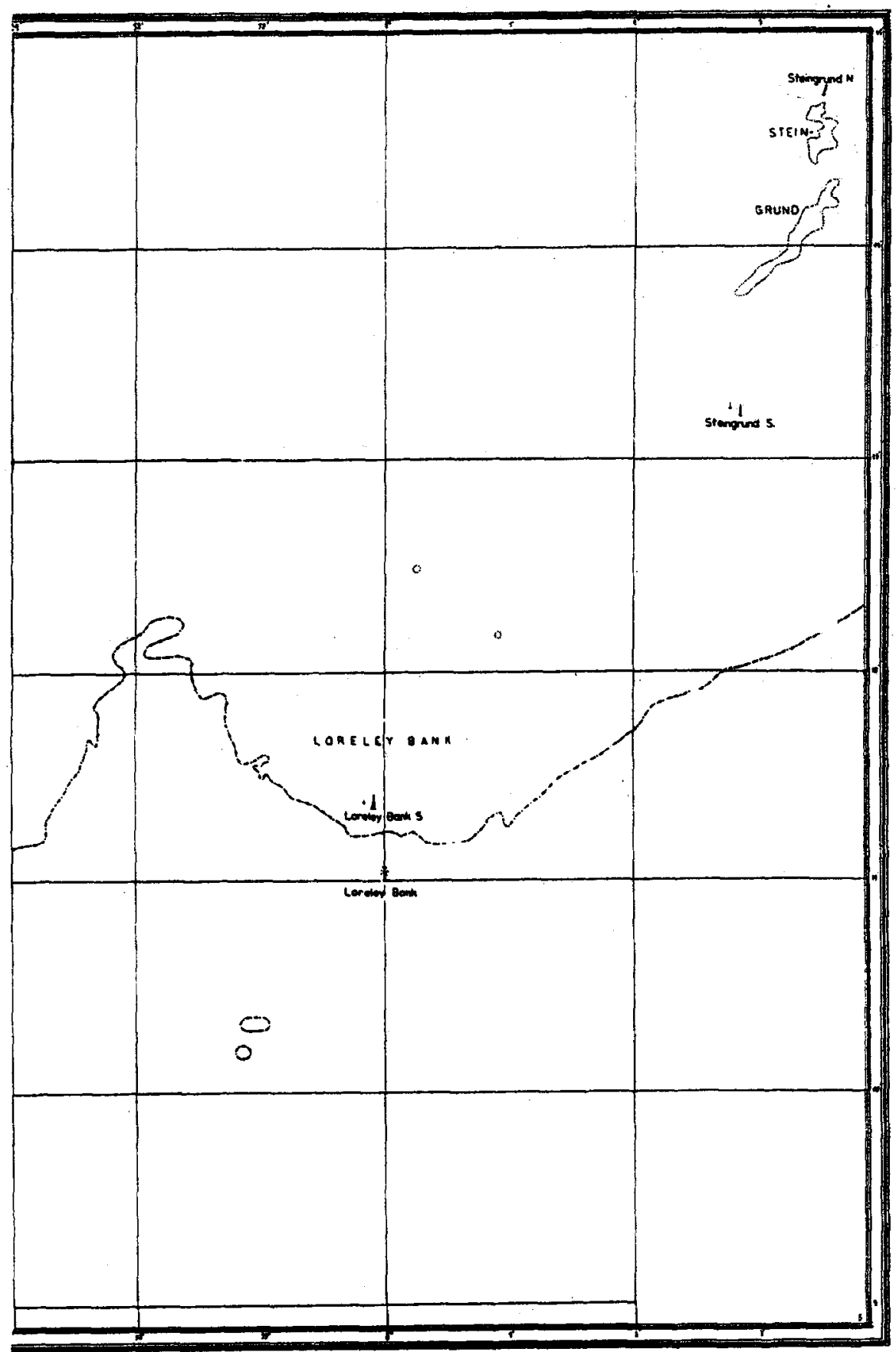


alle vagilen Tiere in die Faunenliste aufgenommen worden. Die Mikrofauna blieb dabei unberücksichtigt.

Die Untersuchung der helgoländer Tonnen geschah 1938-1939; die 1938 aufgeholten untersuchte ich während meines Aufenthaltes auf Helgoland, 1939 hatte Herr Dr. BETH die Freundlichkeit, tierische Proben für mich mitzusammeln. Die Bestimmung der Nadktschnedken und 1 Planarie verdanke ich Dr. H. Herturng $\dagger$ und Prof. J. Meixner $\dagger$.

Das Manuskript war 1940 fertiggestellt; seine Veröffentlichung kann erst jetzt nach dem Kriege erfolgen, wobei die Möglichkeit gegeben war, einige Literaturergänzungen einzufügen. Die Untersuchungen über den Tonnenbewuchs konnte ich 1951-1952 weiterführen durch eine Bearbeitung der Tonnen in der Unterelbe von Hamburg bis ins offene Meer (CASPERs, 1952) als Erweiterung der alten Beobachtungen von KircheNPAUER.

\section{Das Gesamtbild des tierischen Bewuchses Artenliste mit ökologischen Angaben}

Anthozoa

\section{Coelenterata}

Metridium senile (L.)

An mit Miesmuscheln reich besiedelten Tonnen nicht selten in sehr großen Stücken (weiß und rot). Die Seenelken sitzen unter den Mytilus-Kolonien verborgen, wo sie vor zu starken Strömungen geschützt sind. Meist haften sie der Tonne direkt an. Die Besiedlung wird wohl nach dem Ansatz der Muscheln erfolgen, wenn diese etwa $1 \mathrm{~cm}$ groß sind. Kleinere Seenelken sind in einigen Fällen auch zwischen den Amphipoden-Polstern anzutreffen.

Hydrozoa

Tubularia larynx Ellis \& Salander

An einer größeren Zahl von Tonnen (1938: Düne 0/1 und 0/2, Haupt- und Nebentonne, Nathurn, Sellebrunn, Steingrund S-Leuchttonne, 2 Spitzbojen) Überzüge bildend. Bevorzugt werden die tieferen Tonnenteile, wo die Besiedlung von geschützten Winkeln ausgeht. In allen Fällen ist als Mittelpunkt jedes T ubularia-Büschels ein abgestorbener Balanus festzustellen, auf dem der erste Ansatz stattfindet, bis die Stolonen auch auf die Farbe (Giftfarbe, s. BETH) übergreifen. Durch das Zusammenfließen dieser einzelnen Büschel entstehen dann geschlossene Überzüge. Teilweise sind die Kolonien durch aufsitzende andere Hydrozoen, Jassa-Kolonien, MembraniporaUberzüge und Schnecken-Eiballen verschmutzt. Zwischen den Hydranthenstielen wurde Lepidonotus gefunden.

Laomedea flexuosa Alder

Auf einigen Tonnen, deren sonstige Besiedlung recht spärlich war, wurden dithte Rasen festgestellt. Es ist anzunehmen, daß diese Hydrozoen während der ersten Monate der Liegezeit stärker verbreitet sind, jedoch später durch die sonstige Fauna verdrängt werden.

Plumularia setacea (L.)

1939 größere Uberzüge auf 2 Tonnen, besonders Düne 0/2 bildend.

\section{Planaria}

Leptoplana tremellaris O. F. Müller (det. J. MeIXnER) 1 Expl. von Düne 0/2 an der Haltekette zwischen Mytilus. Wie mir Prof. MeIXNer mitteilte, ist die Art vom Mittelmeer, Roten Meer und den atlantischen Küsten Europas bekannt. 


\section{Nemertini}

Prostomatella vermiculus de Quatref. (Prostoma, Tetrastemma)

Düne 0/2. an der Haltekette zwischen Mytilus. Von Helgoland aus der Laminarienregion der Küste bekannt.

Aphroditidae

Polychaeta

Lepidonotus squamatus. (L.)

Nicht selten zwischen Mytilus und Tubularia, z. T. sehr große Würmer.

Phyllodocidae

Anaitides mucosa (Oersted) und $A$. groenlandica (Oersted)

(=Phyllodoce m. u. gr.). Häufig und regelmäßig zwischen den Amphipoden-Polstern.

Nereidae

Nereis pelagica $\mathrm{L}$.

In sehr großen Exemplaren sehr häufig zwischen Mytilus- und Amphipoden-Kolonien.

Serpulidae

Pomatoceros triqueter $\mathrm{L}$.

Meist direkt der Farbe aufsitzend, verhältnismäßig kleine Röhren. Bei einer Oberwucherung durch Amphipoden-Polster usw. sterben die Würmer $a b$, so daß lebende nur an sonst sehr bewuchsarmen Tonnen zu finden sind.

Cirripedia

\section{Crustacea}

Balanus crenatus Brug. und B. improvisus Darw.

Während einige Tonnen ganz frei bleiben, zeigen andere einen sehr dichten Befall mit Seepocken; auch an einer Tonne ist die Größe dabei sehr unterschiedlich, der Durchmesser der Basalplatte von crenatus kann nach einjähriger Liegezeit der Tonnen bis zu 2,2 cm betragen; improvisus erreicht in dieser Zeit nur eine Größe von maximal 1,3 cm. Bei beiden Arten finden sich hohe und flache Wuchsformen, die hohen besonders in gedrängten Kolonien (vgl. hierzu v. Breemen, 1933, S. 248; Neu, 1935 und Caspers, 1949).

Die Gesamthäufigkeit der beiden Arten ist etwa gleich: von 128 bestimmten Stücken gehörten $65 \mathrm{zu}$ improvisus (NEU, $1932 \mathrm{~b}$, hat das Verhältnis von improvisus zu crenatus an helgoländer Tonnen mit 1:20 angegeben). Bei den vermischten Siedlungen herrscht crenatus regelmäßig vor (3:1); ganz rein mit crenatus besiedelte Tonnen sind seltener, nur von improvisus besetzt waren 1938 HSG-Blinktonne, Loreley-Bakentonne und Nathurn. Auf einer Fläche von $400 \mathrm{~cm}^{2}$ wurden bis zu 125 Balanus gezählt (vgl Abb. 9, Düne 0/2, welche die Basalplatten nach Entfernung des MytilusBewuchses zeigt).

Nach v. BREEMEN (1933) ist improvisus im allgemeinen einjährig, doch können einige Exemplare auch ein Alter von etwa zwei Jahren erreichen; die Larvenentwicklung findet in Holland von Mai bis November statt. Für beide Arten wird ein sehr schnelles Wachstum angegeben, wie es auch an den helgoländer Tonnen zu beobachten ist; besonders ist eine Wachstumsbeschleunigung gegenüber $B$. balanoides L. (Runnström, 1925) festzustellen. 
Decapoda

Cancer pagurus $\mathrm{L}$.

Junge Tiere treten gelegentlich zwischen Mytilus auf (Carapaxbreite bis $1,6 \mathrm{~cm})$.

\section{Idothea pelagica Leach}

In größerer Zahl 1938 auf Tonne HK (vor der helgoländer Ostmole) und benachbart auf HKA, also in Strandnähe, zwischen Jassa-Polstern. Wahrscheinlich schwimmen die Asseln meist beim Aufholen der Tonne ab, so daß das Vorkommen auch an anderen Stellen wahrscheinlich ist.

Amphipoda

Jassa pulchella Leach (= Podocerus falcatus Mont.)

Die überall zwischen Algen zu findende Art bildet einen der wichtigsten Bestandteile der Tonnenfauna. Von einigen Individuen, die bei fast älen Tonnen zu finden sind, steigert sich der Befall bis zu 1-2 cm dicken Polstern, die von den Krebsen aus z. T. noch lebenden Algen aufgebaut werden und ein mit Detritus vermischtes dichtes Röhrengeflecht darstellen: Abb. 3. Jeder Amphipode besitzt eine eigene Röhre, aus der er mit dem Vorderende heraussieht (Callame, 1950, gab eine Beschreibung der Röhren). Die Art wurde auch pelagisch beobachtet, doch verlassen die Krebse normaler-

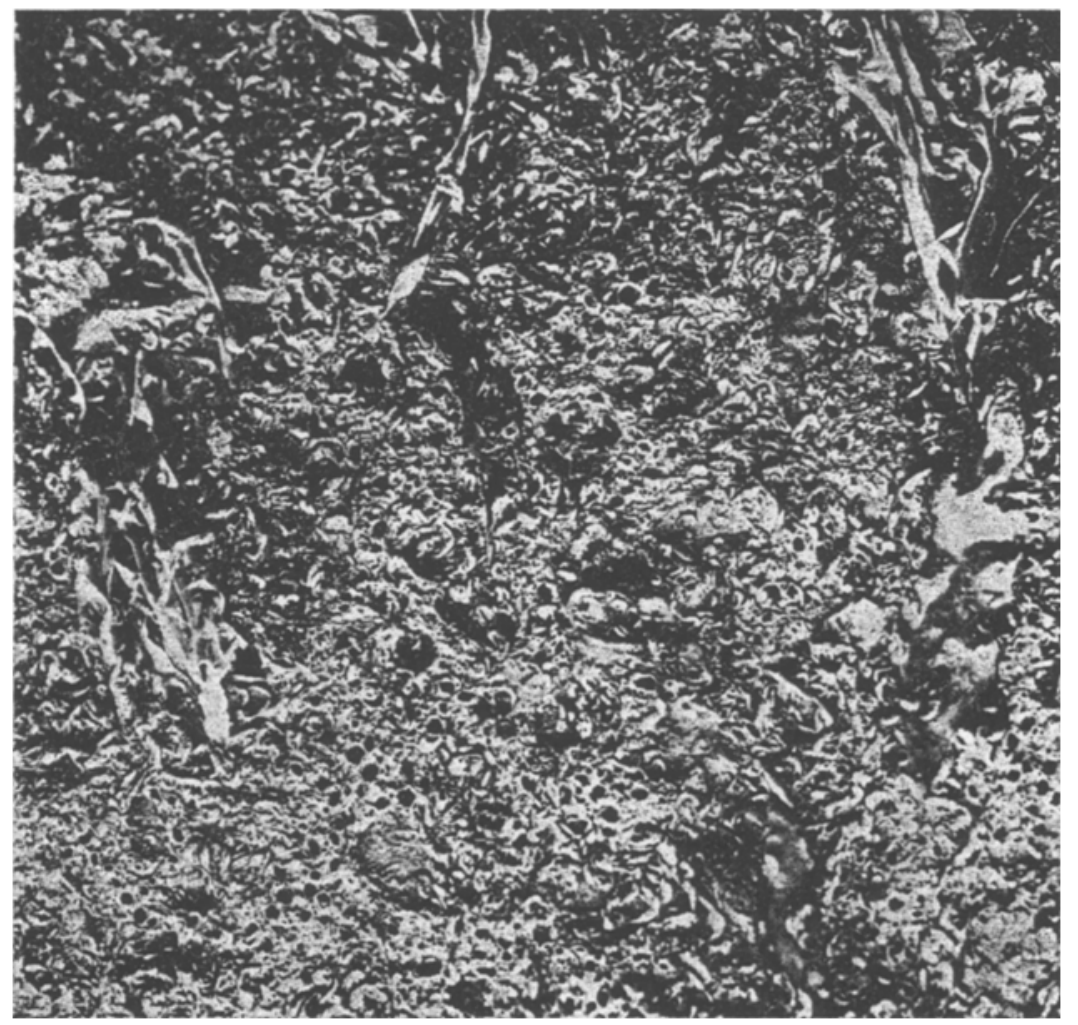

Abb. 3. Amphipoden-Kolonie am Tonnenkörper (Jassa pulchella). Etwa nat. Größe.

weise wohl selten ihre Röhre. Zumeist tritt der Befall der Tonnen bald nach der ersten Entwicklung von fädigen Algen ein; später können die Polster alle vorher angesetzten Balanus, Membranipora usw. überwuchern, 
die jedoch erst bei einer sehr großen Dicke der Polster absterben, während vordem für sie noch die Möglichkeit der Atmung und Nahrungszufuhr gegeben ist. Weibchen mit Eiern wurden besonders im September beobachtet. Die Polster stellen einen Lebensraum für viele vagile Polychaeten usw. dar. Eine Zählung der Anzahl von Amphipoden auf einer bestimmten Fläche ist nicht möglich, da nicht festzustellen ist, wieviele beim Aufholen der Tonne entweichen. Auch eine Schätzung ist schwierig, doch wird die Zahl auf $1 / 10$ qm bei dicken Polstern zwischen 1-3000 liegen. Die Kolonien meiden den obersten Teil der Tonnen unter der Wasseroberfläche, sind sonst aber überall anzutreffen. Eine gleichmäßig glatte Fläche ist Voraussetzung für das Entstehen einer geschlossenen Decke; so finden sich an der Haltekette nur einzelne Krebse zwischen Mytilus.

Gammarus locusta $\mathrm{L}$.

Dieser in der helgoländer Strandregion häufigste Amphipode wurde nur in geringer Zahl auf Tonne HKA 1938 festgestellt. In den Proben von 1939 fehlte er gänzlich.

Gammarellus angulosus (Rathke)

Von dieser zwischen Algen in Küstennähe von Helgoland bekannten Art wurden 1938 einige Exemplare auf Tonne HK 1 gefunden.

Mytilus edulis L.

\section{Lamellibranchia}

Einige Muscheln sind fast an jeder Tonne zu finden; die größeren Kolonien können bei längerer Liegezeit den Tonnenkörper völlig überziehen, wobei nur der oberste Enteromorpha-Streifen freibleibt: Abb. 5 (die kahlen Stellen am unterch Teil des Tonnenkörpers sind durch Abscheuerung beim Aufholen entstanden). Vgl. weiter Abschn. III.

Prosobranchia

\section{Gastropoda}

Lacuna divaricata (Fabr.)

An einigen Tonnen zwischen Algen. Im Juni auch nicht selten die charakteristischen Laichringe.

Opisthobranchia

Facelina drummondi Thomps. (det. H. Hertuing)

7 Stück an der Buhnentonne 3 (Düne) am 13. 9. 1938 zwischen Tubularia.

Membranipora pilosa (L.)

\section{Bryozoa}

Fast an allen Tonnen vertreten: Große Uberzüge direkt auf der Farbe oder häufiger auf allem Bewuchs (Algen, Jassa-Polster, Hydrozoen, Mytilus) z. T. in dichten Krusten. - Andere Bryozoen wurden nie festgestellt.

Asterias rubens L.

\section{Echinodermata}

1938 sehr selten, 1939 in größerer Anzahl an Tonne Düne 0/1 (11.5. 1939) und Blinktonne Steingrund S (11.8.1939); bis $1 \mathrm{~cm}$ Scheibendurchmesser.

Botryllus schlosseri (Pallas)

Tethyodea (Ascidiacea)

Nur an wenigen Tonnen, hier aber große Uberzüge direkt auf der Farbe bildend. 


\section{Der Bewuchs mit Miesmuscheln}

Der erste Ansatz von Mytilus erfolgt an geschützten Stellen, z. B. innerhalb eines abgestorbenen Balanus, zwischen den Kettengliedern, an Eisenwinkeln usw. So zeigt auch der basale Stiel der Boje mit seinen verschiedenen Eisenringen oft einen starken Bewuchs, während der Tonnenkörper ziemlich frei bleibt: Abb. 7, Düne 0/2-Nebentonne und Abb. 4, Steingrund S-Nebentonne. Bei letzterer Tonne ist noch ein Miesmuschelring unterhalb der EnteromorphaZone ausgebildet, wie er auch von FrASER (1938, S. 10) beobachtet worden ist (vgl. S. 156).

Grundsätzlich muß bemerkt werden, daß innerhalb einer Mytilus-Siedlung keine einheitliche Größe der Muscheln besteht: zwischen den großen sind stets kleine vorhanden, die von einem nachträglichen Ansatz von Larven stammen und meist sogar an Zahl die großen überwiegen. Abgestorbene Muscheln fanden sich sehr selten. - Wie auch von Fraser (S. 11) beobachtet, sind die an der Wasserlinie sitzenden Mytilus kleiner als die tieferen, wie aus der in Tabelle 1 wiedergegebenen Messung von der Steingrund S-Nebentonne zu ersehen ist.

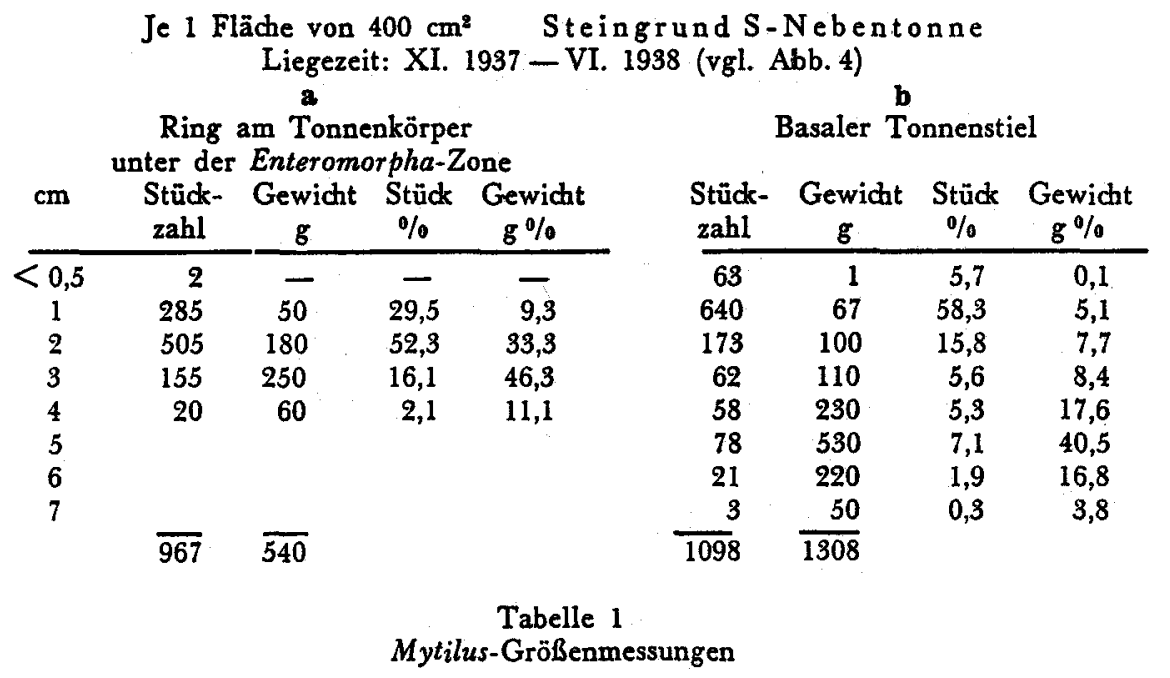

Die höchste Stückzahl haben bei beiden Proben die $2 \mathrm{~cm}$ langen Muscheln, doch zeigt Probe b bei $5 \mathrm{~cm}$ einen Gewichtsanteil von $40 \%$, während die Stückzahl hier nur $7,1 \%$ ausmacht.

Der Größenunterschied ist an den Tonnen bereits bei einem Tiefenunterschied auch von wenigen $\mathrm{cm} \mathrm{zu}$ bemerken: Abb. 8 zeigt die Besiedlung der Tonne Hogstean, wo Mytilus sich im Deckel als dicke Kolonie angesiedelt haben, und zwar hier einseitig, da die Tonne - bedingt durch die Strömung schräg lag, so daß nur die eine Deckelhälfte ständig mit Wasser bedeckt war. An die auch hier ausgebildete Enteromorpha-Zone grenzt zunächst ein etwa $10 \mathrm{~cm}$ breiter Streifen kleinerer Muscheln, an den nach unten zu (hier also zum Deckelrand hin) größere anschließen: Tabelle 2.

Derartige Größenunterschiede sind aber nicht allein durch die verschiedene Tiefenlage gegeben, sondern auch in gleicher Tiefe finden sich vielfach Flecken mit verschiedener Durchschnittsgröße der Muscheln, bedingt durch verschiedene 
Zeitpunkte der Festsetzung. Die Orte, welche die günstigsten Ansatzpunkte bieten (Winkel am basalen Stiel usw.), sind meist von den größten Muscheln besiedelt: Tabelle 3 .

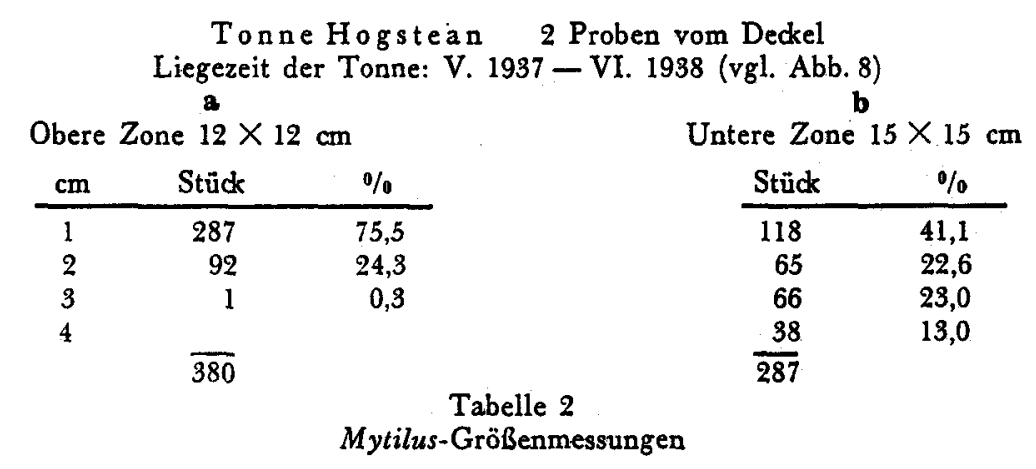

I. Düne $0 / 2-H$ aupttonne

Liegezeit: V. 1937 - VI. 1938 (vgl. Abb. 8)

2 Proben: a) Unterer Teil des Tonnenkörpers, $12 \times 7 \mathrm{~cm}$

b) Von der Kette a

\begin{tabular}{ccccc}
\multicolumn{3}{c}{ Tonnenkörper } & $12 \times 7 \mathrm{~cm}$ & \multicolumn{2}{c}{ Kette } \\
\hline $\mathrm{cm}$ & Stück & \multicolumn{1}{c}{$\%$} & Stück & $\%$ \\
1 & 13 & 16,6 & 1 & 2,3 \\
2 & 37 & 47,3 & - & - \\
3 & 25 & 32,0 & 5 & 11,4 \\
4 & 2 & 2,6 & 14 & 31,8 \\
5 & 1 & 1,7 & 11 & 25,0 \\
6 & & & 13 & 29,5 \\
& 78 & & $\mathbb{4 4}$ &
\end{tabular}

II. Sellebrunn Liegezeit: V. 1937 - VI. 1938 2 Proben: a) Mittlerer Teil des Tonnenkörpers, $8 \times 15 \mathrm{~cm}$

b) Vom Ansatz des basalen Stiels a b Tonnenkörper $8 \times 15 \mathrm{~cm} \quad$ Basaler Stiel

\begin{tabular}{rrrr} 
Stück & $\%$ & Stück & \multicolumn{1}{c}{$\%$} \\
145 & 35,4 & 294 & 50,3 \\
257 & 62,7 & 145 & 24,8 \\
8 & 1,9 & 93 & 15,8 \\
& & 22 & 3,8 \\
& & 26 & 4,5 \\
& & 4 & 0,7 \\
410 & & 584 &
\end{tabular}

Tabelle 3

Mytilus-Größenmessungen

Fraser (S. 11) berichtet, daß die Tonnen der Liverpoul-Bucht auf der Unterseite frei von Mytilus sind. Dies trifft für die helgoländer Tonnen nur bedingt zu: geschlossene Decken fehlen hier, doch sind große Flecken nicht selten (s. Abb. 7). Sehr dicht besiedelt ist auch der innere Hohlraum des basalen Stiels, wenn dieser unten geöffnet ist (bei großen Tonnen).

Aus dem oben Gesagten ergibt sich, daß die Größe der Muscheln in geringerem Umfange von der Tiefenlage, besonders aber von der Zeit des Ansatzes der Larven und damit von der Liegezeit der Tonnen abhängig ist. Die größten Muscheln nach 13monatiger Liegezeit (Mai 1937 bis Juni 1938) maßen 6-7 cm, doch wird dieses Maß auch an Tonnen erreicht, die nur von November 1937 bis Juni 1938, also 8 Monate, draußen waren (vgl. Tabelle 1). Als Hauptwachstumszeit muß danach das Frühjahr angenommen werden. NEU (1933, S. 388) hat an Elbe-Feuerschiff 2 im Juni 1932 nach einer Liegezeit von 11 Monaten die in großer Zahl vorhandenen Mytilus gemessen und fand Muscheln bis zu 6 und 6,5 cm Länge (2 von 3467). Diese Größe führt er auf außerordentlich günstige Ernährungsbedingungen zurück, doch ergibt sich nach den Verhältnissen an den helgoländer Tonnen, daß diese das Normale während dieser Liegezeit darstellt. Die am meisten vorhandenen Muscheln von 

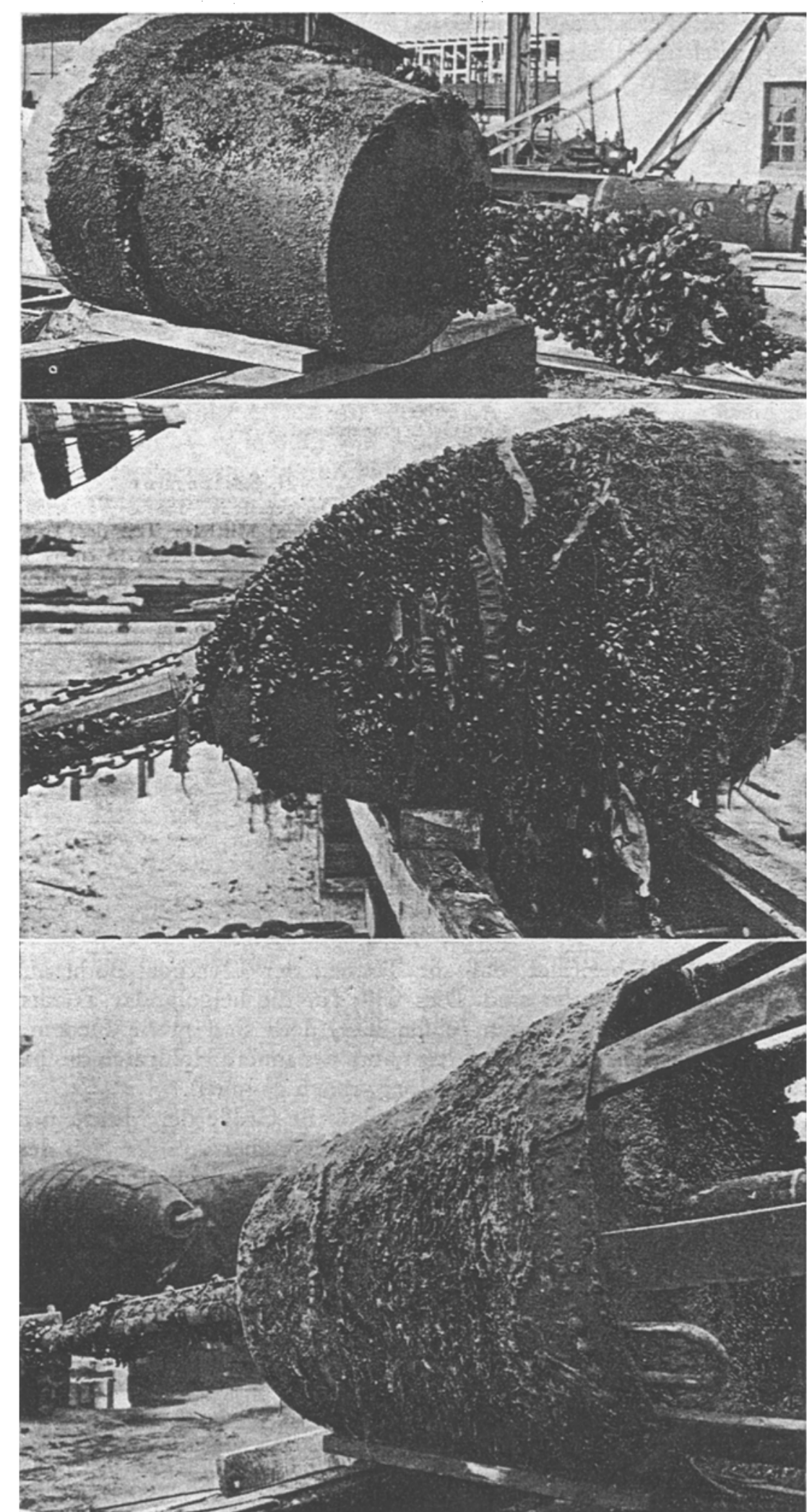
$1-1,5 \mathrm{~cm}$ Länge führt NEU auf eine von Ende April bis Mai reichende Massenansiedlung zurück, während die größeren Muscheln von einer in den Herbstmonaten erfolgten ersten stärkeren Besiedlung stammen sollen. Die im Juni 1938 aufgeholten helgoländer Tonnen zeigten ein ähnliches Bild, doch muß nach den verschiedenen Liegezeiten auf einen anderen Rhythmus geschlossen werden: Die am 25. November 1937 ausgelegte Steingrund S-Nebentonne (s. Tabelle 1) besaß am 2. Juni 1938 bis zu $7 \mathrm{~cm}$ lange Mytilus. Es kann hier nicht angenommen werden, daß diese noch vom Herbstansatz stanmen (auch die in geringem Maße während des ganzen Jahres stattfindende Ansetzung kann nicht die 14,6\% der Stückzahl und 78,7\% des Gewichtes des 4-7 cm großen Muschelanteils verursacht haben), sondern diese werden ebenfalls von der Frühjahrs-Besiedlungsperiode stammen, was wir analog auch für die anderen Proben annehmen müssen. Beweisend ist auch, daß im Juni 1938 ausgelegte und im Dezember des gleichen Jahres wieder eingezogene Tonnen einen nur sehr geringen Ansatz von Muscheln, und zwar nur von kleinen, zeigten. - Nach diesen Ermittlungen hat also Mytilus eine $\mathrm{Haupt-}$ besiedlungszeit im Frühjahr, die sich wahrscheinlich von März bis Mai erstreckt. Demgegenüber spielt der Ansatz zu anderen Jahreszeiten quantitativ eine geringe Rolle.

1. Tonne HSG 2 (s. Abb. 5)

Liegezeit: XI. 1936 - VI. 1938

1 Probe: Unterer Teil des Tonnenkörpérs, $20 \times 20 \mathrm{~cm}$

\begin{tabular}{ccc} 
cm & Stück & $\%$ \\
\hline$<0,5$ & 5 & 1,4 \\
1 & 36 & 9,7 \\
2 & 71 & 19,3 \\
3 & 45 & 12,2 \\
4 & 150 & 40,7 \\
5 & 61 & 16,6 \\
6 & - & -
\end{tabular}

II. Tonne Nathurn Liegezeit: V. 1937 - VI. 1938

2 Proben: a) Mytilus-Fleck, unterer Basalteil des Tonnenkörpers, $20 \times 20 \mathrm{~cm}$

b) Basaler Stiel, Mytilus-Ring, $12 \times 15 \mathrm{~cm}$ Basalteil des Körpers $20 \times 20 \mathrm{~cm}$

\begin{tabular}{cccr}
\multicolumn{2}{c}{$20 \times 20 \mathrm{~cm}$} & \multicolumn{2}{c}{$12 \times 15 \mathrm{~cm}$} \\
Stüdk & $\%$ & \multicolumn{1}{c}{ Stüdk } & \multicolumn{1}{c}{$\%$} \\
\hline- & $\overline{108}$ & $5 \overline{9,0}$ \\
227 & 32,5 & 10 & 5,5 \\
355 & 50,8 & 12 & 6,6 \\
116 & 16,7 & 16 & 8,7 \\
& & 99 & 15,8 \\
& & 8 & 4,4 \\
$\overline{698}$ & & $\overline{183}$ &
\end{tabular}

Tabelle 4

Mytilus-Größenmessungen

Tabelle 4 I zeigt die Größenzusammensetzung von Mytilus an einer Tonne, die 20 Monate im Wasser war (s. Abb. 5). Kennzeichnend ist zunächst die völlige Bedeckung mit Miesmuscheln, unter denen $4 \mathrm{~cm}$ lange am häufigsten sind; besonders große Exemplare sind hier nicht vertreten. Dies spricht dafür, daß der Ansatz erst später erfolgte und dann die vordem hier vorhandene Fauna verdrängte. Die nur mittlere Größe der Muscheln ist aber ferner dadurch bedingt, daß diese in einer derartig dicht besiedelten Kolonie untereinander in starker Nahrungskonkurrenz stehen. Diese wirkt sich für alle

Abb. 4. Nebentonne Steingrund S. Liegezeit: XI 1937-VI 1938. Abb. 5. Tonne HSG 2. Liegezeit: XI 1936-VI 1938. Abb. 6. Tonne Hogstean. Liegezeit: V 1937-VI 1938. 
gleichmäßig aus, so daß nicht einige im Wachstum stark vorauseilen können, wie dies an anderen Stellen (Eisenringe usw.) möglich ist. Bei letzteren ist dann auch eine stärkere Aufspaltung der Größengruppen gegeben. Zeit der Ansetzung und Besiedlungsdichte wirken hier zusammen.

Als Beispiel für die Besiedlungsdichte sind in Tabelle 4 II noch 2 Zählungen von der Leuchttonne Nathurn aufgenommen, die zusammen mit den quantitativen Angaben der übrigen Tabellen ein Bild von der Zahl auf verschiedenen Flächen geben. Natürlich ist die Stückzahl abhängig von der Größe der Muscheln: auf einer Fläche von $400 \mathrm{~cm}^{2}$ (vgl. Tabelle 1 a u. b, 4 I u. 4 II a) schwankt die Zahl zwischen $1098(1-2 \mathrm{~cm}: 74,1 \%)$ und $368(3-5 \mathrm{~cm}$ : $69,5 \%)$.

\section{Der Besiedlungsvorgang}

Aus der verschiedenen Liegezeit der Tonnen und dem Bewuchsaufbau beim Aufholen kann ein Bild von dem zeitlichen Verlauf der Besiedlung gewonnen werden. Die Mehrzahl der Tonnen wird im Juni-Juli ausgewechselt, während der Herbstmonate ist also noch genügend Zeit zum Ansatz der ersten Tiere. Als erste tierische Besiedler überhaupt (abgesehen von Mikroorganismen) sind wohl die Seepocken anzusehen. Bald darauf, nach dem Wachstum der Algenkeime, finden sich auch Amphipoden (Jassa) ein, die aber zunächst noch keine größeren Kolonien bilden. Bis zu diesem Stadium ist die Besiedlung fast bei allen Tonnen einheitlich. Die weitere Entwicklung des Bewuchses ist dann von der Lage der Tonne abhängig (vgl. Abschn. VI). Während des Winters findet nur eine geringe Neuansiedlung statt, so daß die eigentliche Massenentwicklung im Frühjahr anzusetzen ist, wie dies bereits für Mytilus (Abschnitt III) dargestellt wurde. Im Herbst kann es entsprechend der stärkeren Entwicklung der Algen allerdings noch zu einer größeren Ausbildung von Jassa-Kolonien kommen. Der stärkste Bewuchs findet im Frühjahr durch die Miesmuscheln statt. Auf den von ihnen eingenommenen Flächen kommt es zu einer weitgehenden Verdrängung der bisherigen Fauna: so sterben die Seepocken, die zuerst als Kristallisationspunkte der Mytilus-Ansiedlung dienten, zum größten Teil ab; ebenso werden Algen (außer Laminarien) vernichtet. Dieser Vorgang ist auch von TRUsherm (1932) beobachtet worden (s. ferner CASPERS, 1949); derartige Uberwachsungen nennt Richter (1922) einen „in sich bedingten Faunenwechsel“" (vgl. Abschn. V). Nur eine dicke Lage von Jassa-Polstern scheint für den Mytilus-Befall ein Hemmnis zu sein; auch Muscheln, die sich bereits hier angesiedelt hatten, erreichten nur eine geringe Größe.

Durch die stärkere Ausbildung des Bewuchses ist auch der Lebensraum für die vagilen Tiere gegeben (vgl. Abschn. V). Bei einer Liegezeit über mehrere Jahre würde Mytilus wohl bei allen Tonnen das Übergewicht bekommen, so daß sich später ein geschlossener Muschelmantel ausbildet (vgl. Tonne HSG 2, Abb. 5).

Dieser normale zeitliche Verlauf der Besiedlung kann aber beträchtliche Unterschiede aufweisen, auf die in Abschnitt VI näher eingegangen werden soll. 


\section{Die Lebensgemeinschaft}

Die zunächst als „tabula rasa “ im Wasser schwimmenden Tonnen geben die seltene Gelegenheit, das Entstehen einer isolierten Lebensgemeinschaft zu verfolgen. Der allgemeine Gang der Besiedlung ist im vorigen Abschnitt geschildert worden, so daß hier nur noch die Frage nach der ökologischen Stellung dieser Biozönose behandelt zu werden braucht.

Für den Biotop ist zunächst kennzeichnend, daß er einen nur sehr kleinen Raum bildet, der allseitig von Wasser umgeben ist. Das Wasser mit seinen Verschiedenheiten der Planktonzufuhr hat den größten Einfluß auf das Bild der Tonnen-Gemeinschaft (vgl. auch Abschn. VI); dagegen spielt der Untergrund nur eine Rolle als Festsetzungsfaktor ${ }^{3}$ ).

Es ergibt sich nun die Frage, ob die Biozönose der Tonnen einer anderen bekannten des Meeresbodens gleichzusetzen ist. Die Verhältnisse des Biotops haben Ahnlichkeit mit denen an flachen Meeresstellen auf festem Untergrund, am meisten mit dem auch durch die benachbarte Lage einen Vergleich herausfordernden helgoländer Felssockel. In den flachen Gebieten muß hier aber der Gezeitenfaktor berücksichtigt werden, der sich in Trockenliegeperioden, im ganzen - auch bei den ständig untergetauchten Gemeinschaften - im Unterschied der Höhe der Wasserbedeckung geltend macht. Der Wegfall dieses Faktors ist aber gerade für die Tonnen ein charakteristisches Merkmal (abgesehen von der durch die Gezeiten hervorgerufenen, an den Tonnen vorbeiflutenden Gezeitenströmung). Bedingt hierdurch fehlt auch beim tierischen Tonnenbewuchs vertikal eine zonale Gliederung, wie sie sonst an festen Gegenständen in der Gezeitenzone zu beobachten ist (außer dem durch kleinere Muscheln ausgezeichneten Mytilus-Saum unterhalb der Enteromorpha-Zone; s. Abschn. III. Uber Algenzonen s. BETH, 1952). Die im Felswatt vertretene obere Zone von Balanus balanoides fehlt daher an den Tonnen ${ }^{4}$ ).

Zum Vergleich müssen wir also ein nicht mehr von dem Gezeitenwechsel betroffenes Gebiet wählen, wie wir es in den tieferen Teilen des helgoländer Felssockels antreffen. Uber dieses Gebiet sind wir noch mangelhaft unterrichtet, da es für eine Untersuchung schwer zugänglich ist, doch reichen unsere bisherigen Kenntnisse (vgl. HAgmeier, 1930 a) aus, um zu sagen, daß eine Parallelität zwischen der dortigen Fauna und der der Tonnen nich t besteht: im Felsgebiet fehlen die Mytilus- und Jassa-Kolonien; letztere müßten ja entsprechend auch hier wenigstens in einigen Teilen größere UUberzüge auf den Felsen bilden. Umgekehrt sind hier natürlich viele Arten vertreten, die auf den Tonnen fehlen, wofür 3 Gründe anzugeben sind: 1. ist das Vorkommen einiger Arten im Felsgebiet durch die relativ größere Tiefenlage (bei Flut) bedingt, 2. sind bei der auf den Tonnen gegebenen kurzen Entwicklungszeit noch nicht alle Arten hierhin gelangt, deren Vorkommen denkbar wäre, 3. wird aber die Entwicklung dieser Arten auf den Tonnen auch dadurch verhindert,

3) Durch die Giftwirkung der verschiedenen Anstriche gegebene Unterschiede sind in der vorliegenden Darstellung nicht behandelt. Die Farbe wirkt auch nur während des Ansatzes der Larven, kann also die Ausbildung des Bewuchses verzögern, aber nicht gänzlich unterbinden. Immerhin kann durch die Hinausschiebung des Larvenansatzes ein Unterschied der Erstbesiedlung und damit eine Veränderung in der biologischen Konkurrenz statthaben; direkte Beobachtungen hierüber an den Tonnen sind schwierig; KüHL (1949) macht hierzu Angaben beim Hafenbewuchs.

4) Die Art ist aber in Küstennähe an schwimmenden Planken beobachtet worden (NEU, 1932 a, S. 107). 
daß einige Tierarten sich in kurzer Zeit über die zum Ansatz verfügbare Fläche verbreiten und für später sich ansetzende Arten eine starke Platz- und Nahrungskonkurrenz bedeuten. Auf Punkt 3 muß zum größten Teil die Artenarmut auf den Tonnen zurückgeführt werden, die auch schon KIRCHENPAUER (S. 5) aufgefallen ist. Diese ist sonst ökologisch das Zeichen eines „extremen Lebensraumes", dem nur wenige Organismen, diese aber in großer Individuenzahl, angepaßt sind (vgl. Thienemann, S. 7). Als ein solcher können die Tonnen aber nicht aufgefaßt werden; im Gegenteil sind die Lebensbedingungen an einem im Wasser schwebenden festen Gegenstand als optimal für die meisten festsitzenden Tiere zu bezeichnen. Auch die isolierte Lage kann für den tierischen Bewuchs nicht als Hindernis wirken, da die Zufuhr ansatzreifer Larven durch die Strömung gewährleistet ist (vgl. hierzu aber Abschn. VI). Umgekehrt ist der Wellenschlag nicht so stark wie an Küstengebieten.

Der hauptsächlich für das Erscheinungsbild der Biozönose nach einjähriger Liegezeit der Tonnen verantwortliche Faktor ist die Kürze der Zeit, die es noch nicht zu einer Ausgeglichenheit der Lebensgemeinschaft hat kommen lassen, nachdem die Tonnen anfänglich völlig organismenfrei waren.

Diese Unausgeglichenheit des Tonnenbewuchses zeigt auch der oft weitgehende Wechsel der Besiedlung an einer Tonne im Verlaufe von 1-2 Jahren. Wohl bieten bereits die ersten sich ansetzenden Balanus zusammen mit jungen Algen und einigen Amphipoden das Bild einer typischen Vergesellschaftung, ebenso wie die später sich bildenden Jassa-Polster; alle haben aber den Charakter des Unfertigen, es fehlt der labile Gleichgewichtszustand einer Lebensgemeinschaft. Erst die am Ende sich ausbildenden Miesmuschelkolonien zeigen konstantere Verhältnisse.

Wir kommen zu der Auffassung, daß der Tonnenbewuchs eine Biozönose darstellt, deren Bildungsstadien uns in typischen Sukzessionen entgegentreten. An den Tonnen können wir also die Sukzessionsfolge (Thienemann, 1939, S. 8, Tischler, 1949, 1950) einer solchen, durch die festsitzenden Formen geprägten Lebensgemeinschaft studieren. Nach den vorliegenden Untersuchungen scheint das Klimaxsta dium bei den helgoländer Tonnen in den meisten Fällen eine MytilusKolonie zu sein (vgl. Abschn. IV), welche hier „die natürliche Lebensgemeinschaft der Lebensstätte" (Tischler, 1949) darstellt, nur daß die sich entwickelnde und dem Klimaxstadium zustrebende Biozönose vor dem Erreichen dieses Endstadiums fast regelmäßig vom Menschen vernichtet wird $\mathrm{d}^{5}$ ).

Auf dem Lande haben wir eine ungefähr parallele Entwidklung und dann folgende Vernichtung einer Biozönose beim Kahlschlagsystem von Wäldern, wie sie auf Fichtenkahlschlägen in montaner Lage untersucht worden ist (LeITINGER-MICOLETZKY, 1940).

Im ganzen haben wir es also mit einem sukzessiven Ablösen von Artenkombinationen zu tun (TISCHLER, 1951), deren Entwicklung dabei gerichtet verläuft, so daß man gewisse Voraussagen treffen kann, von einer Pionierbesiedlung über die Folgestadien zum Klimaxstadium, wobei die einzelnen Sukzessionen reversibel sind $\left.{ }^{6}\right)$. Bei den Tonnen sind während der Entwicklungszeit des Bewuchses gleichzeitig auf engstem Raum nebeneinanderliegende Siedlungen vorhanden (vgl. Abschn. VI), die verschiedenen Sukzessionsstadien

5) Es wäre interessant, bei Gelegenheit Tonnen zu untersuchen, die sich bereits viele Jahre im Wasser befinden.

6) Für Sukzessionen in Landbiotopen vgl. hierzu JANETscheK (1948/49). 
entsprechen und auch die oben erwähnte Unausgeglichenheit der Gemeinschaft zeigen.

Durch die jahreszeitlichen Unterschiede in der Zufuhr der ansatzreifen Larven kann bei einer verschiedenen Auslegezeit der Tonnen die Aufeinanderfolge der Sukzessionen geändert werden, ohne jedoch wohl den Gesamtverlauf und das Endstadium grundlegend zu beeinflussen (s. auch Anm. 3).

An manchen Tonnen ist auch zu beobachten, daß die normale Entwicklung der Besiedlung an einigen Stellen plötzlich dadurch abgebrochen wird, daß die Farbschicht abblättert. Die neue Oberfläche bildet wieder eine "tabula rasa", und es ist bemerkenswert, daß nicht in kurzer Zeit der Bewuchs der benachbarten unversehrten Flächen hier übergreift, sondern daß sich ein gänzlich neuer Bewuchs bildet, der Úbereinstimmung mit dem ersten Bewuchs an Tonnen zeigt. So hoben sich 1938 an der Tonne Nathurn zwischen Amphipoden-Kolonien Flecken ab, die dicht mit - an den übrigen Teilen der Tonne fehlenden - Hydrozoen (und kleinen Algen) besiedelt waren.

Bei einer längeren Entwidklungszeit der Biozönose würden sich auch noch mehr vagile Tiere als "Endobiose" einfinden und entwickeln (besonders zeitweise schwimmende Polychaeten, Isopoden u. a.); ebenso würden die Epizoen auf den Algen und Tieren eine artliche Bereicherung erfahren. Auch in dieser Hinsicht ist also die Gemeinschaft bei der zur Verfügung stehenden Zeit „ungesättigt".

Bei dieser Besprechung der Eigenart der Biozönose müssen den Tieren noch die Algen hinzugefügt werden. Immerhin will mir scheinen - wenn eine solche Wertung überhaupt berechtigt ist -, daß der Algenbewuchs nicht die bestimmende Rolle im Bild dieser Lebensgemeinschaft hat, wie es für einige Elemente des tierischen Bewuchses zutrifft. Wohl ist einige Zeit vor und nach dem Balaniden-Ansatz ein Algenrasen die Regel, doch wird dieser in den größten Teilen der Tonne später durch die Jassa- und Mytilus-Kolonien verdrängt, wobei vorwiegend die fädigen Algen zum Aufbau der Röhren verwendet werden. Nur die Laminarien haben bei älteren Tonnen quantitativ einen großen Anteil, doch ist ihre Bindung an den Biotop nur durch die im Verhältnis kleine Haftwurzel gegeben, während der Thallus sonst frei im Wasser flutet.

\section{Die regionale Verteilung}

Nachdem in den beiden vorhergehenden Abschnitten der normale Gang der tierischen Tonnenbesiedlung ökologisch behandelt wurde, ist es jetzt noch unsere Aufgabe, regionale Unterschiede des Tonnenbewuchses zu untersuchen, auf die bereits mehrfach hingewiesen wurde. Vor allem ergibt sich die Frage, ob Gesetzmäßigkeiten bestehen, wobei 1. regionale Unterschiede an einer Tonne und 2. Unterschiede zwischen den einzelnen Tonnen behandelt werden müssen.

Die Unterschiede an einer Tonne sind besonders durch die MytilusVerteilung augenscheinlich (vgl. Abschn. III und Abb. 4 bis 8), doch zeigen auch die Jassa-Polster, Hydrozoen-Kolonien usw. Fleckbildungen; ferner gibt es besiedlungsfreie Stellen neben dichten Tier-(und Pflanzen-)zonen. In vielen Fällen sind diese Erscheinungen auf besondere morphologische Eigenarten der Tonne (Winkel, Unterseite des Tonnenkörpers usw.) zurückzuführen; die ge- 


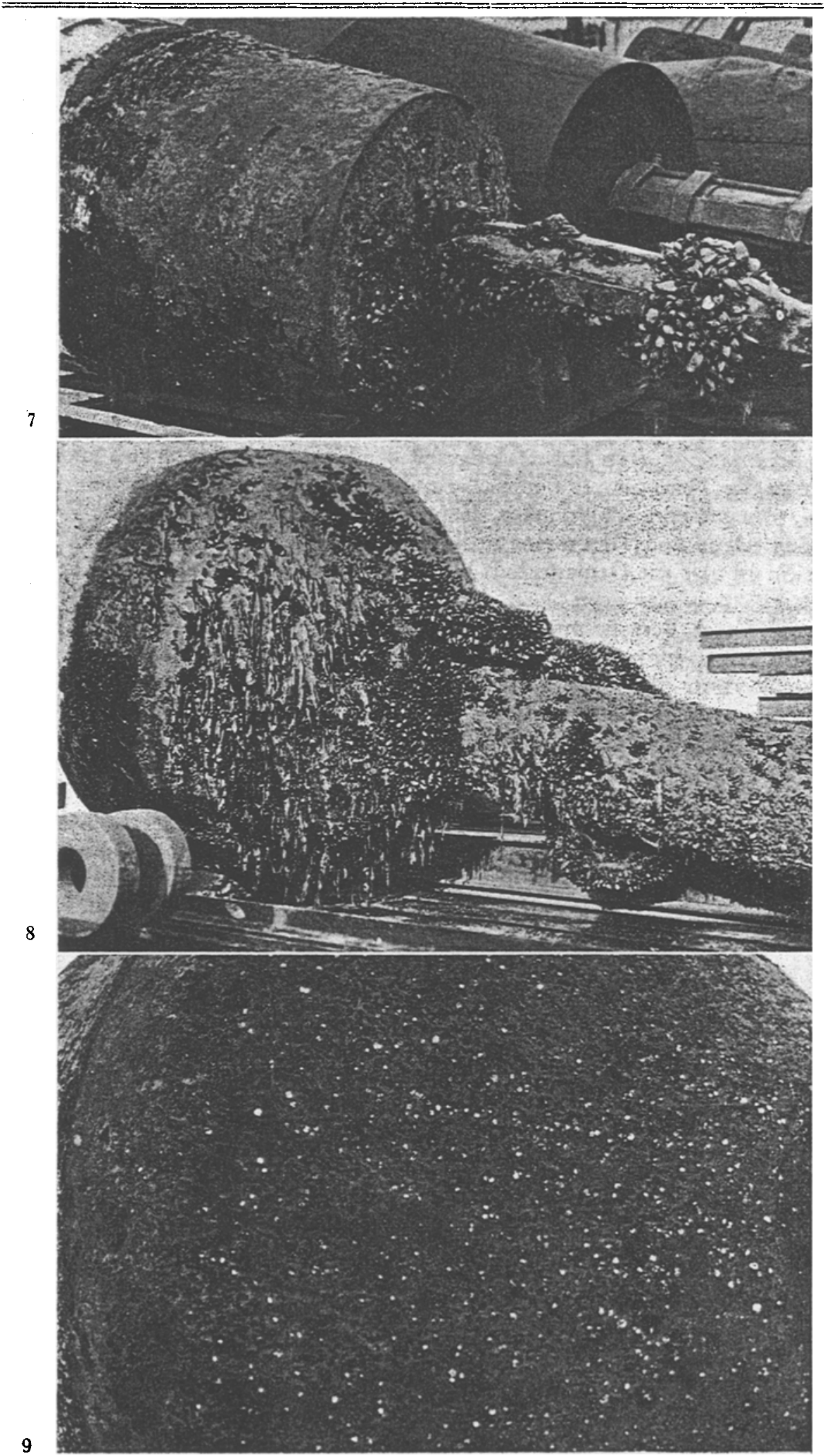


schütztesten Ansatzpunkte werden vorwiegend von Miesmuscheln eingenommen (vgl. die Abbildungen). Für eine ganze Reihe von Fleckbildungen ist jedoch keine durch die Morphologie des Untergrundes gegebene Ursache erkennbar. Auch Seitenunterschiede (bei durch die Strömung ständig schräg liegenden Tonnen) sind gelegentlich zu beobachten, ohne daß doch hieraus eine Gesetzmäßigkeit abgelesen werden könnte.

Der Bewuchs verschiedener Tonnen weist noch größere Unterschiede auf: von sehr stark besiedelten Tonnen gibt es alle Zwischenstufen bis zu fast völlig kahlen (nach einjähriger Liegezeit); von letzteren sei die Tonne Steingrund N 1938 genannt, die neben einem ganz geringen Algenansatz (im unteren Teil fast ausschließlich Diatomeen) nur 15 lebende Balanus und 1 kleine Mytilus als Bewuchs aufwies.

Es wurden auf Grund der Untersuchungen viele Karten über die Verbreitung der einzelnen Arten auf den verschiedenen Tonnen angefertigt, um eine Gesetzmäßigkeit der regionalen Verteilung zu ermitteln, jedoch ohne jeden Erfolg! Weder für die inselnahen im Gegensatz zu den inselfernen, noch für die in einer Himmelsrichtung liegenden Tonnen war irgendein Zusammenhang festzustellen. Ebenso kann sich der tierische Bewuchs der gleichen Tonne (mit demselben Anstrich!) in zwei aufeinander folgenden Jahren (1938 und 1939) gänzlich unterscheiden.

In der Algenbesiedlung von Tonnen mit gleicher Liegezeit sind nach den Ermittlungen von BETH (1952) deutliche regionale Unterschiede in bezug a uf den Abstand vonder Insel festzustellen. Dieser Unterschied zur tierischen Besiedlung beruht wahrscheinlich auf der wesentlich kürzeren Lebensdauer der Algensporen im Vergleich zu den Larvenstadien der Tiere.

Diese Regellosigkeit wird besonders augenscheinlich, wenn man zwei unmittelbar nebeneinander liegende Tonnen (Haupt- und Nebentonne) vergleicht.

Als Beispiel sei ein Vergleich der Haupt- und Nebentonne Düne $0 / 2$ gegeben (vgl. Abb. 7 u. 8). Gegenüber dem sehr starken Bewuchs der Haupttonne mit Mytilus (und Laminarien) ist der Gesamtbewuchs an der Nebentonne arm: am Tonnenkörper breiten sich hier stellenweise Jassa-Kolonien aus, doch sind auch gänzlich besiedlungsfreie Flecken vorhanden. Amphipoden bilden bei der Haupttonne am basalen Stiel größere Polster, zwischen denen sich nur kleine Mytilus-Fledken finden. Tubularia-Büschel waren auf beiden Tonnen häufig. Zwischen den dicken Miesmuschelkolonien der Haupttonne fanden sich: Metridium senile. Cancer pagurus, Nereis pelagica, Lepidonotus squamatus und Leptoplana tremellaris, alles Arten, die auf der Nebentonne wegen der geringen Entfaltung der Miesmuscheln fehlen. Seepocken sind auf beiden Tonnen vorhanden, gehäuft jedoch auf der Haupttonne, und hier in der Region der dichtesten Miesmuschel-Verbreitung, wo auf einer Fläche von $30 \times 30 \mathrm{~cm}$ 110 Stück gezählt wurden, von denen 12 noch lebten: vgl. Abb. 9, die die Balanus-Basalplatten nach Abkratzen der Muscheln zeigt.

Aus den Beobachtungen ergibt sich die Frage, ob alle Verschiedenheiten des Bewuchses - $z$ wischen einzelnen Tonnen und in Form von Besiedlungsflecken an einer Tonne - auf morphologische und sonstige Gegebenheiten des Tonnenuntergrundes zurückzuführen sind oder ob auch Differenzierungen in der Zufuhr ansatzreifer Larven der für die verschiedenen Sukzessionen kennzeichnenden Arten eine Rolle spielen. Wenn auch, wie oben dargestellt, zweifellos Winkelbildungen usw. an der Tonnengestalt für das Bewuchsbild von größter Bedeutung sind, so drängt sich angesichts der Bilder der Besied-

Abb. 7. Nebentonne Düne 0/2. Liegezeit: V 1937-VI 1938.

Abb. 8. Haupttonne Düne 0/2. Liegezeit: V 1937-VI 1938.

Abb. 9. Haupttonne Düne 0/2. Balanus-Basalplatten nach Entfernung des Mytilus-Bewuchses. Untere Hälfte des Tonnenkörpers. 
lungsflecken innerhalb einer zumindest erkennbar morphologisch nicht differenzierten Fläche der Tonnenoberfläche die Vermutung auf, daß diese auf enger begrenzte Larvenballungen während der planktischen Phase zurückgehen. Manche "Regellosigkeit" der Bewuchsverteilung sowohl an einer Tonne als auch regional zwischen den einzelnen Tonnen würde hiermit eine befriedigende Erklärung finden: treten die Larven wenigstens zeitweise in räumlich beschränkten Verbänden auf, so braucht nur ein kleiner Teil der Tonnenoberfläche damit in Berührung zu kommen, um eine Fledkbildung des Bewuchses auf dieser $\mathrm{zu}$ ergeben; eine dicht daneben liegende Tonne, an der sonst die gleichen Besiedlungsbedingungen herrschen, bleibt völlig frei von diesen Larven, falls sie außerhalb der Grenze des Larvenverbandes liegt. Auch vertikale Zonenbildungen könnten auf diese Verhältnisse zurückgeführt werden, z. B. bei der Nebentonne Steingrund S der obere Mytilus-Ring (Abb. 4), der von Larven herrühren wird, die im Wasser eine dünne Oberflächenschicht einnahmen.

Mir will es scheinen, daß dieser zusätzlichen kausalen Deutung der Fleckbildung durch Larvenballungen im Plankton neben den übrigen nachgewiesenen Faktoren eine Bedeutung zukommt. Es muß aber betont werden, daß es für die Vermutung eines auf kleinerem bis kleinstem Raum beschränkten Larvenverbandes keine exakten planktologischen Unterlagen gibt. Vielleicht könnten hierauf einmal Beobachtungen gerichtet werden.

Die in der Nordsee festgestellten isolierten Wasserkörper mit höherem Salzgehalt, die eine Zeitlang als Blase innerhalb salzärmeren Wassers bestehen (s. KüNNE, 1937, u. CASPERS, 1938, S. 15), nehmen einen größeren Raum ein, als er für die Erklärung der Besiedlungserscheinungen auf den Tonnen gefordert werden muß $B^{7}$ ).

Eine Schwierigkeit für die planktologische Feststellung solcher Larvenverbände ist, daß zwischen ansatzreifen und noch nicht soweit entwickelten Larven unterschieden werden müßte. Die Ansatzreife ist wahrscheinlich auf eine kurze Zeit beschränkt, und die in der Umgebung sich findenden Larven, die noch nicht ansatzreif sind, rufen bei der Berührung mit einer Tonne noch keinerlei Bewuchs hervor $\left.{ }^{8}\right)$. Ferner werden die Verhältnisse durch Stromwirbel an der Tonne differenziert. Wesentlich ist wohl auch die Tidenzeit, da wahrscheinlich während des Kenterns des Stromes der Hauptansatz stattfindet. Die Größe der hypothetischen Larvenverbände wird sehr verschieden sein. Aus den Bewuchsbeobachtungen möchte man vermuten, daß sie minimal bis zu einer Gruppe weniger Larven heruntergehen, die dann z. B. einen kleinen Balanus- oder Bryozoen-Fleck hervorrufen. Umgekehrt zeigt der gleichmäßige Befall benachbarter Tonnen mit einer Art (z. B. Haupttonne und Nebentonne Düne 0!2: Tubularia), daß der Larvenverband größer als die Entfernung der Tonnen war (abgesehen von der Möglichkeit, daß der Befall auch auf mehrere Larvenschübe zurückgehen kann).

Mytilus-Flecke brauchen nicht immer auf solchen Larvenballungen zu beruhen, da (abgesehen von der Ansetzung in Winkeln usw.; vgl. oben) die Ab-

7) Dies trifft ebenso für Flecken dichtester Besiedlung - besonders von Muscheln am Meeresboden zu, deren Entstehung von Davis (1923 u. 1925) und Hagmeier (1930 b) durch eine örtlich besonders reiche Zufuhr von Larven während eines Jahres erklärt wird (vgl. auch CAspers, 1938, S. 85)

8) Ansatzbereite Larven vermögen fernerhin in bezug auf den Festsetzungsort eine gewisse Auswahl zu treffen, wie es für die Cyprislarven von Balaniden festgestellt worden ist (Visscher, 1930, Pomrat u. Weiss, 1946, Weiss, 1946, 1947, Küht, 1950); sie schwimmen of tmals sogar wieder los, um sich anderswo neu anzusetzen. 
hängigkeit auch indirekt sein kann, wie bei dem oben gegebenen Beispiel der Besiedlung der Haupt- und Nebentonne Düne 0/2: die Haupttonne (Abb. 8) wies an der schrägen Unterseite des Körpers eine dicke geschlossene Miesmuscheldecke auf. Nach Abkratzen dieser fanden sich hier sehr viele - meist abgestorbene - Balanus (Abb. 9). Die Fleckbildung war hier also zunächst durch die Seepocken gegeben, die später Ansatzpunkte für Mytilus wurden und die Bildung der Muscheldecke an diesen Stellen hervorriefen. So gehen auch an anderen Tonnen oft einzelne kleine Mytilus-Flecke und TubulariaBüschel auf einzelne Seepocken zurück, die als erster Ansatzpunkt dienten.

Das Fehlen einer Art auf einer Tonne kann endlich dadurch bedingt sein, daß die später die Tonne erreichenden ansatzreifen Larven durch die hier bereits vorhandene Fauna an der Ansiedlung gehindert werden (Platz- und Nahrungskonkurrenz).

\section{Vergleich mit dem Schiffsbewuchs}

Bereits von Hentschel (1923, 1925) wurde der Schiffsbewuchs als eine eigene Lebensgemeinschaft aufgefaßt; die Berechtigung hierzu ist von NEU (1932 c) in einem Aufsatz zusammenfassend dargestellt worden. Wie alle Autoren betonen, stellt der Schiffsbewuchs eine sehr starke Auslese dar, gegeben durch die extremen Bedingungen bei der Fahrt, während die eigentliche Bildung des Bewuchses auf die Hafenliegezeit zurückgehit (s. áuch KüHL, 1940). Die während der Fahrt einsetzende Entsiedelung und der Neubewuchs in zum Teil weit entfernten Häfen stellen Verhältnisse dar, die von denen an verankerten Tonnen gänzlich verschieden sind. Der Charakter der Lebensgemeinschaft muß aber bei Tonnen und fahrenden Schiffen als gleich bezeichnet werden: beide stellen nur verschiedene, durch die Umweltsbedingungen gegebene Variationen dar.

Artliche und morphologische Unterschiede zwischen dem Bewuchs an Schiffen und Tonnen greifen ineinander. Die Möglichkeit einer größeren Artenfülle bei fahrenden Schiffen ist durch den Besuch verschiedener Häfen bedingt; umgekehrt bewirkt der Ortswechsel mit den dadurch gegebenen Anderungen von Salzgehalt, Temperatur usw., daß nur wenige Arten sich halten können; entscheidend ist besonders die Strömung, welche zartere Tiere (und besonders Pflanzen) bald völlig abscheuert. Ferner ist hierdurch eine Gestaltsänderung der am Leben bleibenden Tiere (z. B. Balanus) gegeben. Diese Auslese bewirkt auch, daß sich nicht ein größeres Organismengeflecht bilden kann, welches für vagile Tiere einen Lebensraum bieten könnte. So sind die großen Mytilusund Amphipoden-Kolonien ein Kennzeichen der Tonnen. Speziell an die Bedingungen an fahrenden Schiffen angepaßte Tiere (Balaniden, Serpuliden, Membranipora) können hier aber eine stärkere Entwicklung als an den Tonnen haben, wo sich die biologische Konkurrenz anderer Tiere geltend macht.

Tonnen- und Schiffsbewuchs stellen Variationen einer Lebensgemeinschaft dar. An den Tonnen können die verschiedenen Sukzessionen der Gemeinschaft untersucht werden, deren weitere Ausbildung an den Schiffen durch die extremen Bedingungen während der Fahrt, die eine weitgehende Auslese und Reduktion zur Folge haben, verhindert wird. Der Bewuchs an Tonnen steht unter optimalen Lebensbedingungen, die eine verstärkte biologische Konkurrenz während der verschiedenen Erscheinungsbilder bei der Entwicklung der Lebensgemeinschaft bewirken. 


\section{Zusammenfassung}

Die Liste des tierischen Bewuchses an helgoländer Seetonnen (1938-1939) enthält 22 Arten, von denen 9 festsitzend sind. Den wichtigsten Bewuchs bilden Seepocken, Miesmuscheln und Amphipoden-Kolonien (Jassa pulchella). Unter den Hydrozoen sind Tubularia und Laomedea häufig. Die Polychaeten (außer Serpuliden) bilden kennzeichnende Bewohner der Mytilus- und Jassa-Kolonien. Von Balanus sind zwei Arten vertreten: B. crenatus und B. improvisus (Gesamtverhältnis $1: 1$; in gemischten Siedlungen überwiegt $B$. crenatus $3: 1$ ).

Der erste Ansatz von Mytilus erfolgt an geschützten Stellen nach der Balaniden-Ansiedlung. Altere Tonnen können völlig mit Muscheln überzogen sein. Die Größe nach einjähriger Liegezeit der Tonne beträgt bis $7 \mathrm{~cm}$, doch wird diese Größe wohl nach dem für die Massenentwicklung ausschlaggebenden Frühjahrsbrutfall (März bis Mai) in einer Zeit von 4-5 Monaten erreicht. An der Wasseroberfläche sitzende Muscheln sind kleiner als die tieferen, ferner sind die Größenunterschiede durch den Zeitpunkt der Ansetzung und die Gunst des Festsetzungspunktes gegeben. Abhängig von der Größe schwankt die Zahl auf einer Fläche von $400 \mathrm{~cm}^{2}$ zwischen $1100(1-2 \mathrm{~cm}: 74 \%)$ und $370(3-5 \mathrm{~cm}$ : $70 \%)$.

Der Besiedlungsvorgang nach dem Auswechseln der Tonnen im Juni-Juli geht normalerweise so vor sich, daß sich zunächst Seepocken ansetzen; bald finden sich Amphipoden ein. Im Frühjahr erfolgt stellenweise ein Massenansatz von Miesmuscheln, ferner bilden sich $-z$. T. schon im Herbst - große Amphipodenpolster aus, die für den Mytilus-Befall ein Hemmnis bedeuten. Die Miesmuscheln bringen die ihnen oft als erste Ansatzpunkte dienenden Balanus zum größten Teil zum Absterben.

Die Lebensgemeinschaft an den Tonnen enthält Elemente aus der Gezeitenzone der felsigen Küste, weist jedoch durch den Ausfall der Gezeiten und die Kürze der gegebenen Entwidklungszeit andere Verhältnisse auf. Für die Biozönose ist die Unausgeglichenheit kennzeichnend: jedem der untereinander sehr verschiedenen Stadien fehlt das stabile Gleichgewicht. Das Endstadium, in den meisten Fällen wohl eine Mytilus-Kolonie, wird durch das vorherige Aufholen der Tonnen selten erreicht. Die verschiedenen, zeitlich aufeinander folgenden oder zum Teil nebeneinander vorkommenden $\mathrm{Er}$ scheinungsbilder in der Entwicklung der Biozönose stellen Sukzessionen dar, die in ihrer Folge in Abhängigkeit von der Auslagezeit der Tonne variieren können, im ganzen aber dem Klimaxstadium der Miesmuschelkolonie zustreben.

Die Besiedlung an einer Tonne ist außerordentlich verschiedenartig; ebenso weisen die einzelnen Tonnen untereinander - auch zwei an einer Stelle liegende - große Unterschiede auf, die keinerlei Gesetzmäßigkeit der Tierverbreitung nach regionalen Gesichtspunkten erkennen lassen. Das gleiche trifft für den Bewuchs in aufeinanderfolgenden Jahren zu. Einmal gehen die Besiedlungsflecken auf morphologische und sonstige Eigenarten des Tonnenuntergrundes zurück; es wird aber vermutet, daß ein Teil der Flecken auch auf Ballungen ansatzreifer Larven im Plankton beruht, so daß diese also in regional beschränkten Verbänden auftreten und infolgedessen nur jeweils eine Tonne oder eine kleine Fläche dieser erfaßt wird. Mytilus-Flecken können später indirekt durch die fleckenartige Verbreitung der als Ansatzpunkte oft dienenden Balaniden entstehen. Der planktologische Beweis für die Annahme räumlich zum Teil eng begrenzter Planktonballungen fehlt. 
Der Schiffsbewuchs stellt eine Variation der gleichen Lebensgemeinschaft wie die der. Tonnen dar; sie wird durch die extremen, eine weitgehende Auslese und Reduktion bewirkenden Verhältnisse bei der Fahrt geprägt. Die Lebensgemeinschaft der Tonnen steht unter optimalen Lebensbedingungen, die eine verstärkte Konkurrenz der Arten während der verschiedenen Entwidklungsphasen der Lebensgemeinschaft zur Folge haben.

\section{Schriftenverzeichnis}

Beth, K., 1952: Der Algenbewuchs an helgoländer Seetonnen. Helgol. wiss. Meeresunters. 4. Breemen, L. van, 1933: Zur Biologie von Balanus improvisus Darwin. Zool. Anz. 105.

Callame, B., 1950: Observations sur le nid de l'Amphipode Jassa falcata (Montagu). C. r. Acad. Sci. (Paris) 231.

Caspers, H., 1938: Die Bodenfauna der helgoländer Tiefen Rinne. Helgol. wiss. Meeresunters. $2,1$.

- 1941: Der tierische Befall am Holz der helgoländer Seebrücke. Zool. Anz. 136, 12.

- 1949: Die Bewuchsgemeinschaft an der Landungsbrücke der Nordseeinsel Spiekeroag und das Formproblem von Balanus. Zool. Jahrb. (Syst.) 78, 3.

- 1952: Der Bewuchs auf den Fahrwassertonnen im Süß-, Bradk- und Meerwasserbereich der Unterelbe. Ein quantitativ-biozönotischer Vergleich (Manuskript).

Coe, W. R., 1932: Season of attachment and rate of growth of sedentary marine organisms at the pier of the Scripps Institution of Oceanographie, La Jolla, California. Bull Scripps Inst. Oceanogr. Univ. of California, La Jolla, Calif. Techn. Ser. 3.

Davis, F. M., 1923, 1925: Quantitative Studies on the Fauna of the Sea Bottom. Nr. 1. Preliminary Investigation of the Dogger Bank. Nr. 2. - Results of the Investigations in the Southern North Sea 1921-24. Fishery Invest. London II 6, 2 und 8, 4.

Fraser, J. H., 1938: The Fauna of fixed and floating structures in the Mersey Estuary and Liverpool Bay. Proc. Liverpool Biol. Soc. 51.

Hagmeier, A., 1930 a: Die Besiedlung des Felsstrandes und der Klippen von Helgoland. Teil I. Der Lebensraum. Wiss. Meeresunters. N. F. Abt. Helg. 15.

- 1930 b: Eine Fluktuation von Mactra (Spisula) subtruncata da Costa an der ostfriesischen Küste. Ber. Dtsch. wiss. Komm. Meeresforsh. N. F. 5.

Hentschel, E., 1923: Der Bewuchs an Seeschiffen. Int. Revue ges. Hydrobiol. u. Hydrogr. 11.

- 1924: Das Werden und Vergehen des Bewuchses an Schiffen. Mitt. Zool. Staatsinst. u. Zool. Museum Hamburg 41.

Janetschek, H., 1948/49: Tierische Sukzessionen auf hochalpinem Neuland. Ber. Naturw. Med. Ver. Innsbruck 48/49.

Kirchenpauer, G. H., 1862: Die Seetonnen der Elbmündung. Abh. a. d. Gebiete d. Naturw., herausg. v. Natw. Ver. Hamburg, 4.

Kühl, H., 1946. Über die Beziehungen zwischen Fahrtgebiet und Schiffsbewuchs. Probl. d. Schiffsantriebes, München.

- 1949. Uber die Wirkung von Kontaktinsektiziden in Bewuchsschutzanstrichen. Verh. Deutsch. Zoologen, Kiel, 1948.

- 1950: Über die normale und die durch Gifte beeinflußte Metamorphose von Balanus improvisus (Darwin). Verh. Deutsch. Zool., Mainz, 1949.

- 1951: Vergleichende biologische Untersuchungen über den Hafenbewuchs. Verh. Deutsch. Zool. Ges., Marburg, 1950.

Künne, Cl., 1937: Über die Verbreitung der Leitformen des Großplanktons in der südlichen Nordsee im Winter. Ber. Dtsch. wiss. Komm. Meeresforsch., N. F. 8.

Leitinger-Micoletzky, E., 1940: Die Tiersukzession auf Fichtenschlägen. Zool. Jahrb. (Syst.) 73.

Milne, A., 1940: The ecology of the Tamar Estuary IV. The distribution of the fauna and flora on buoys. J. Mar. Biol. Ass. U. K. 24.

$\mathrm{Neu}, \mathrm{W}, 1932$ a: Untersuchungen über den Schiffsbewuchs. Int. Revue ges. Hydrobiol. u. Hydrogr. 27.

- 1932 b: Das Vorkommen von Balanus improvisus Darwin auf helgoländer Seetonnen. Zool. Anz. 99.

- 1932 c: Der Schiffsbewuchs als Lebensgemeinschaft. „Der Naturforscher“ 9. 
- 1933 a: Qualitative und quantitative Bewuchsuntersuchungen am Elbe-Feuerschiff „Bürger meister Bartels" ${ }^{*}$ 1932. Arch. Hydrobiol. 25.

- 1933 b: Biologische Arbeiten über den Schiffsbewuchs. Int. Revue ges. Hydrobiol. u. Hydrogr. 29.

- 1935: Mitteilung einiger Beobachtungen zur Formbildung von Balanus balanoides L. und dessen Ansiedlung. Zool. Anz. 110.

Orton, J. H., 1930: Growth-inhibitive value of paints. J. Mar. Biol. Ass. U. K. 16.

Pomrat, C. M. u. C. M. We iss, 1946: The influence of texture and composition of the surface on the attachment of sedentary organisms. Biol. Bull. 91, 1.

Pyefinch, K. A., 1948: Notes on the Biology of Cirripeds. J. Mar. Biol. Ass. U. K. 27.

R i chter, R., 1922: Flachseebeobachtungen: in sich bedingter Faunenwechsel. Sendkenbergiana 4.

Runnström, S., 1925: Zur Biologie und Entwidklung von Balanus balanoides (L.). Bergens Museum Aarbok; Naturvidensk. raekke 5.

Thienemann, A., 1939: Grundzüge einer allgemeinen Okologie. Arch. Hydrobiol. 35.

Tischler, W., 1949: Grundzüge der terrestrischen Tierökologie. Braunschweig, Fr. Vieiweg \& Sohn.

- 1951: Zur Synthese biozönotischer Forschung. Acta Biotheoretica 9, 4.

Trusheim, F., 1932: Paläontologisch Bemerkenswertes aus der Okologie rezenter NordseeBalaniden. Senckenbergiana 14.

Visscher, J. P., 1930: Nature und Extent of Fouling of Ship's Bottom, 1928. Bull. Bureau of Fisheries, Washington, 43, Part II.

We iss, Ch. M., 1947: The effect of illumination and stage of tide on the attachment of barnacle cyprids. Biol. Bull. 93, 3.

- 1948: Seasonal and annual variations in the attachment and survival of barnacle cyprids. Biol. Bull. 94, 3.

Anschrift des Verfassers: Dozent Dr. H. Caspers, Zoologisches Staatsinstitut, Hamburg 36, Jungiusstr. 6 . 